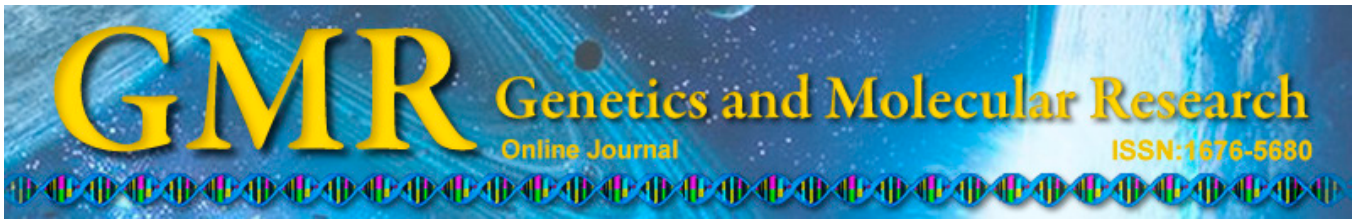

\title{
Vascular endothelial growth factor gene is associated with hypertensive cerebellar hemorrhage and rehabilitative treatment
}

\author{
Q.S. He ${ }^{1}$, L.F. Yang ${ }^{1}$, W.B. Wang ${ }^{1}$, B. Yuan ${ }^{2}$, L.Y. Zhang ${ }^{3}$ and X.J. Guo ${ }^{1}$ \\ ${ }^{1}$ Department of Rehabilitation Medicine, \\ The First Affiliated Hospital of Xinxiang Medical University, \\ Xinxiang, Henan, China \\ ${ }^{2}$ Department of Internal Neurology, \\ The First Affiliated Hospital of Xinxiang Medical University, \\ Xinxiang, Henan, China \\ ${ }^{3}$ Department of Rheumatology Nephropathy, \\ The First Affiliated Hospital of Xinxiang Medical University, \\ Xinxiang, Henan, China \\ Corresponding author: Q.S. He \\ E-mail: heqingsongys@126.com
}

Genet. Mol. Res. 14 (3): 9849-9857 (2015)

Received December 4, 2014

Accepted May 6, 2015

Published August 19, 2015

DOI http://dx.doi.org/10.4238/2015.August.19.18

\begin{abstract}
Early rehabilitative therapy is important for patients with hypertensive cerebral hemorrhage to improve long-term function of the extremities. Vascular endothelial growth factor (VEGF) is closely associated with the pathogenesis of hypertension. To identify the markers contributing to the genetic susceptibility to hypertensive cerebellar hemorrhage $(\mathrm{HCH})$ and rehabilitative treatment, we examined the potential association between $\mathrm{HCH}$ and 12 single nucleotide polymorphisms of the $V E G F$ gene. Participants included 244 patients with $\mathrm{HCH}$ and 251 healthy controls from our rehabilitation department. The $\mathrm{T}$ allelic frequencies of the rs3025020 (intron 6) and rs3025039 (3'-UTR) polymorphisms were significantly higher in the patients with
\end{abstract}


$\mathrm{HCH}$ than in the healthy controls (rs3025020 T allele: $\mathrm{P}=0.0002, \mathrm{OR}$ $=1.619,95 \% \mathrm{CI}=1.256-2.088 ;$ rs $3025039 \mathrm{~T}$ allele: $\mathrm{P}=0.001, \mathrm{OR}$ $=1.682,95 \% \mathrm{CI}=1.246-2.270)$. Strong linkage disequilibrium was observed in three blocks ( $\mathrm{D}^{\prime}>0.9$ ), and significantly more C-G-C (rs3025020, rs3025030, and rs3025039) haplotypes $(\mathrm{P}=0.001)$ were found in the controls in block 3 . Significantly more T-G-C haplotypes were found in the patients with HCH $(\mathrm{P}=0.046)$. Further genotype and clinical phenotype correlation study of the rs3025039 carriers showed that Fugl-Meyer and Barthel index scores were lower in the patients with the TT genotype relative to $\mathrm{CT}+\mathrm{CC}$ genotypes $(\mathrm{P}<0.01)$. These findings point to a role for $V E G F$ polymorphism in $\mathrm{HCH}$, and may be informative for future investigations on the pathogenesis of rehabilitative treatment.

Key words: Rehabilitative treatment; Hypertensive cerebellar hemorrhage; Vascular endothelial growth factor; Single nucleotide polymorphisms

\section{INTRODUCTION}

Hypertensive cerebral hemorrhage $(\mathrm{HCH})$ is one of the most serious complications in hypertension. The onset of $\mathrm{HCH}$ is rapid, and its recovery is very slow, resulting in high mortality and disability rates. Early rehabilitative therapy is important for the patients with $\mathrm{HCH}$ to improve long-term function of the extremities. This early intervention can prevent a series of complications such as bedsores, respiratory and urinary infections, deep phlebitis, and joint contracture and deformity. It can also reduce the incidence and development of spastic paralysis, a classic sequelae in hemiplegia, in the flexors of upper extremities, and in the extensors of lower extremities. Furthermore, it helps maintain the range of motion in the joint, and recover the limb function.

Vascular endothelial growth factor (VEGF), a specific mitogen and survival factor for endothelial cells, is a crucial promoter of angiogenesis in physiological and pathological conditions (Ferrara and Davis-Smyth, 1997). It is generally accepted that VEGF is a hypotensive mediator (Rini et al., 2011). When blood pressure is elevated, VEGF is expressed to antagonize this elevation (Schrijvers et al., 2004; Advani et al., 2007). VEGF has been reported to be a predictive marker of the hypertension response to target therapy with drugs such as sunitinib, sorafenib, and bevacizumab (Dahlberg et al., 2010; Jain et al., 2010; De Stefano et al., 2011; Rini et al., 2011). Hypertension is an important risk factor for atherosclerotic disorders, and may be a consequence of structural alterations in the microvascular network, resulting partly from abnormal regulation of VEGF (Celletti et al., 2001; Loomans et al., 2004). Belgore et al. (2001) demonstrated that plasma VEGF-A levels were significantly raised compared with normotensive controls, but significantly reduced after the treatment of hypertension. Collectively, these data suggest that the $V E G F$ gene is an excellent target for the treatment of hypertension.

Human $V E G F$, located on the chromosome 6, has 8 exons and 7 introns, and is typically expressed as a $46-\mathrm{kDa}$ homodimer. A number of single nucleotide polymorphisms (SNPs) have been found in VEGF, including rs699947 and rs1570360 in the promoter (Brogan et al., 1999), rs3025020 in intron 6 (Blumberg et al., 2008), rs2010963 in the 5'-untranslated region (5'-UTR) (Lee et al., 2010), and rs3025039 in the 3'-UTR (Renner et al., 2000). All of these 
SNPs have been reported to be associated with VEGF protein expression. It has been reported that rs2010963 was associated with hypertension (Hamedian et al., 2012), whereas rs699947 $\mathrm{C} / \mathrm{C}$ genotype was associated with a lower incidence of grade 3 or 4 hypertension (Schneider et al., 2008). However, only a few loci (1-4 SNPs) were analyzed in these studies, resulting in significant discrepancies between the studies. A limited number of SNPs could not effectively capture the true causative SNPs in the VEGF gene due to the weak linkage disequilibrium between them. Thus, more SNPs should be investigated to effectively capture the true causative SNPs in the VEGF gene. Furthermore, to our knowledge, the association of VEGF polymorphisms with $\mathrm{HCH}$ and rehabilitative treatment has not been examined.

In this study, to examine the association of VEGF polymorphisms with $\mathrm{HCH}$ and rehabilitative treatment, we examined the potential association between HCH and 12 SNPs (rs699947, rs 1570360, rs2010963, rs833069, rs3024997, rs30249998, rs3025000, rs3025006, rs3025010, rs3025020, rs3025030, and rs3025039) of the VEGF gene using the MassARRAY system.

\section{SUBJECTS AND METHODS}

\section{Subjects}

This case-control study enrolled 244 unrelated subjects with $\mathrm{HCH}$ (mean $\pm \mathrm{SD}$ age: $62 \pm 5.61$ years). The patients were hospitalized in our rehabilitation department between May 2008 and July 2014. All patients underwent standard neurological and clinical examinations, as well as routine laboratory tests. The diagnosis of $\mathrm{HCH}$ was performed by computed tomography $(\mathrm{CT})$. Some patients underwent $\mathrm{CT}$ angiography or magnetic resonance imaging to exclude aneurysm rupture, cavernous angioma, arteriovenous malformation, tumor, or hemorrhagic infarction.

The control group consisted of 251 unrelated healthy subjects (mean \pm SD age: 61.6 \pm 6.5 years) who underwent health examinations in the Medical Examination Center of the First Affiliated Hospital of the Xinxiang Medical College (Xinxiang, China). All participants were from a non-genetically related Chinese Han population in He'nan Province (China). The study was performed according to the Guidelines of the Medical Ethics Committee of Xinxiang Medical College (Xinxiang, China). Written informed consent was obtained from all the participants recruited in this study.

\section{Treatment}

The groups of patients received conventional medications after surgery. Forty-eight hours after the vital signs were stabilized and no neurological symptoms progressed, the patients in the observation group received the post-operative rehabilitative therapy, including early passive exercise in bed. The therapy used the Bobath concepts as its core, and was gradually performed according to the staging of the Bobath therapy and disorders of cerebral hemorrhage. The therapy included correct position in bed (correct limb position), in-bed exercise, sit-stand training, balancing exercise in sitting position, balancing exercised in standing position, walking exercise, language exercise, and self-care ability exercise. Four weeks after the therapy, the evaluation was performed using the Fugl-Meyer scoring method (physical functions) and the Barthel index scoring method (self-care ability). 


\section{Evaluation criteria}

A motor score $<50$ points indicated severe motor disorder; $50-84$ points motor disorder; 85-95 points moderate motor disorder, 96-99 points mild motor disorder, and 100 points normal motor functions. We also used muscular strength to evaluate the subjects. The evaluation criteria were described as follows: fundamental recovery: general restoration of motor functions of the limbs, the upper limbs were able to perform delicate movements, and the lower limbs were capable of walking; significant response: the paralyzed limbs were significantly improved, with muscular strength improved to grade II or above; moderate response: paralyzed limbs were moderately improved, and muscular strength was improved to grade I-II; no response: no significant change in muscular strength after treatment. Criteria for efficacy evaluation in aphasia: excellent efficacy was defined as being able to communicate in language, with clear articulation that was as good as that before disease onset; good efficacy was defined as being able to have simple conversations, with clear articulation, and words were in logical order; moderate efficacy was defined as being unable to communicate effectively but being able to pronounce single words; poor efficacy was defined as being unable to pronounce words even after the training mentioned above.

\section{Genotyping}

Three to five milliliters of peripheral blood was collected in tubes coated with EDTA. Genomic DNA was extracted from the leukocytes in the blood using the EZNA ${ }^{\mathrm{TM}}$ Blood DNA Midi Kit (Omega Bio-Tek, Norcross, GA, USA), according to the manufacturer protocol. Primers for PCR and single-base extension were designed by using the Assay Designer software package (Sequenom Inc., San Diego, CA, USA). SNP genotyping was performed by Shanghai Benegene Biotechnology Co., Ltd. (Shanghai, China) using the MassARRAY system (Sequenom) by means of matrix-assisted laser desorption ionization-time of flight mass spectrometry method (MALDI-TOF) according to manufacturer instructions. The completed genotyping reactions were spotted onto a 384-well spectroCHIP (Sequenom) using the MassARRAY Nanodispenser (Sequenom) and determined by MALDI-TOF. Genotype calling was performed in real-time with the MassARRAY RT software version 3.0.0.4 and analyzed using the MassARRAY Typer software version 3.4 (Sequenom).

\section{Statistical analysis}

All statistical analyses were carried out using the SPSS 16.0 software (SPSS Inc., Chicago, IL, USA). Hardy-Weinberg equilibrium and associations between the case-control status and each polymorphism were assessed by the Pearson chi-square test. Bonferroni's correction was used to adjust the test level when multiple comparisons were conducted, and the $\mathrm{P}$ value was divided by the total number of loci. Pairwise linkage disequilibrium statistics $\left(\mathrm{D}^{\prime}\right.$ and $\mathrm{r}^{2}$ ) and haplotype frequencies were computed using Haploview 4.0 to construct haplotype blocks. Haplotype blocks were defined according to the 'solid spine of linkage disequilibrium (LD)' approach. Haplotypes with a frequency of less than 5\% were excluded. The frequency of all haplotypes was compared between the case and control participants using the Pearson $\chi^{2}$ test. 


\section{RESULTS}

There was no significant deviation from Hardy-Weinberg equilibrium for any SNP in patients with $\mathrm{HCH}$ and the healthy controls. The genotype distributions, allelic frequencies, and haplotypes in patients with $\mathrm{HCH}$ and the healthy controls are shown in Tables 1-4. The Fugl-Meyer and Barthel index scores after treatment by VEGF gene rs3025020 and rs3025039 were shown in Table 5. LD analyses revealed 3 haplotypes in controls, and also showed that 3 SNPs (rs699947, rs1570360, and rs2010963) were located in block 1, 6 SNPs (rs833069, rs3024997, rs30249998, rs3025000, rs3025006, and rs3025010) were located in block 2, and 3 SNPs (rs3025020, rs3025030, and rs3025039) were located in block 3 (Figure 1).

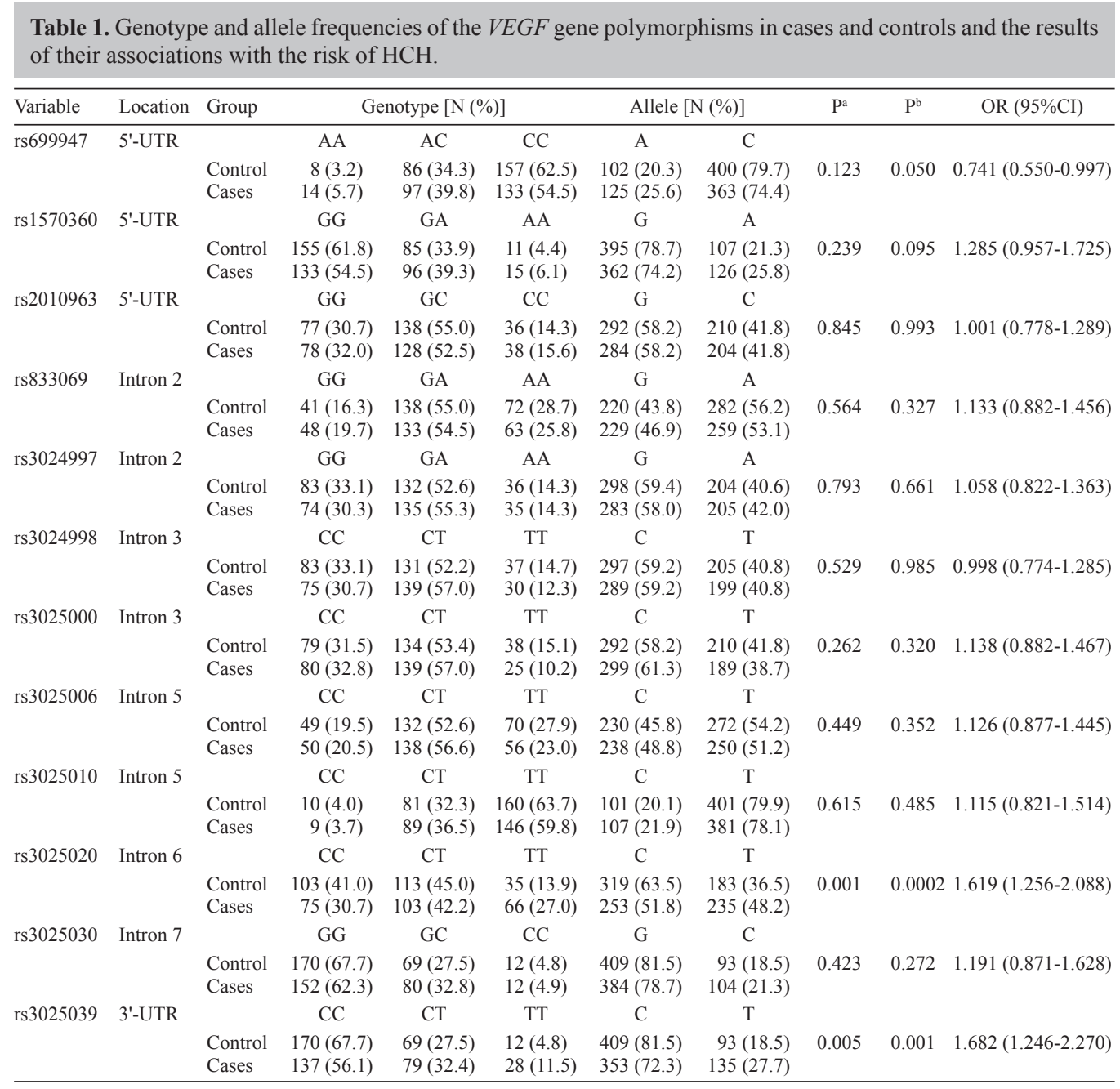

${ }^{\mathrm{a} P}$ values for genotype frequency difference. ${ }^{\mathrm{b}} \mathrm{P}$ values for allele frequency difference. 
Table 2. VEGF haplotype in block 1 frequencies and the results of their associations with risk of $\mathrm{HCH}$.

\begin{tabular}{lcccccc}
\hline Haplotype $^{\mathrm{a}}$ & Cases [N (\%)] & Controls [N (\%)] & \multicolumn{4}{c}{ Statistics } \\
\cline { 4 - 6 } & & & $\chi^{2}$ & $\mathrm{P}$ & OR & $95 \%$ CI \\
\hline C-G-C & $89(36.5)$ & $105(41.8)$ & 1.490 & 0.222 & 0.798 & $0.556-1.146$ \\
C-G-G & $83(34.0)$ & $92(36.7)$ & 0.376 & 0.540 & 0.891 & $0.616-1.288$ \\
A-A-G & $51(20.9)$ & $50(19.9)$ & 0.073 & 0.787 & 1.062 & $0.686-1.645$ \\
\hline
\end{tabular}

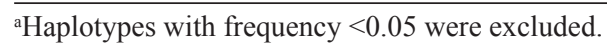

Table 3. VEGF haplotype in block 2 frequencies and the results of their associations with risk of $\mathrm{HCH}$.

\begin{tabular}{|c|c|c|c|c|c|c|}
\hline \multirow[t]{2}{*}{ Haplotype $^{a}$} & \multirow[t]{2}{*}{ Cases [N (\%)] } & \multirow[t]{2}{*}{ Controls [N (\%)] } & \multicolumn{4}{|c|}{ Statistics } \\
\hline & & & $\chi^{2}$ & $\mathrm{P}$ & OR & $95 \% \mathrm{CI}$ \\
\hline G-A-T-T-T-T & $90(36.9)$ & $93(37.0)$ & 0.001 & 0.969 & 0.993 & $0.689-1.430$ \\
\hline $\mathrm{A}-\mathrm{G}-\mathrm{C}-\mathrm{C}-\mathrm{C}-\mathrm{T}$ & $56(23.0)$ & $60(23.9)$ & 0.063 & 0.802 & 0.948 & $0.625-1.438$ \\
\hline $\mathrm{A}-\mathrm{G}-\mathrm{C}-\mathrm{C}-\mathrm{C}-\mathrm{C}$ & $46(18.9)$ & $49(19.5)$ & 0.036 & 0.850 & 0.958 & $0.612-1.498$ \\
\hline A-G-C-C-T-T & $25(10.2)$ & $25(9.9)$ & 0.036 & 0.850 & 0.958 & $0.612-1.498$ \\
\hline
\end{tabular}

${ }^{a}$ Haplotypes with frequency $<0.05$ were excluded.

Table 4. VEGF haplotype in block 3 frequencies and the results of their associations with risk of HCH.

\begin{tabular}{|c|c|c|c|c|c|c|}
\hline \multirow[t]{2}{*}{ Haplotype $^{\mathrm{a}}$} & \multirow[t]{2}{*}{ Cases [N (\%)] } & \multirow[t]{2}{*}{ Controls [N (\%)] } & \multicolumn{4}{|c|}{ Statistics } \\
\hline & & & $\chi^{2}$ & $\mathrm{P}$ & OR & $95 \% \mathrm{CI}$ \\
\hline C-G-C & $76(31.1)$ & $113(45.0)$ & 10.088 & 0.001 & 0.552 & $0.382-0.798$ \\
\hline C-C-T & $35(14.3)$ & $46(18.3)$ & 1.434 & 0.231 & 0.746 & $0.462-1.206$ \\
\hline $\mathrm{T}-\mathrm{G}-\mathrm{C}$ & $110(45.1)$ & $91(36.3)$ & 3.997 & 0.046 & 1.443 & $1.007-2.069$ \\
\hline
\end{tabular}

${ }^{\mathrm{a}}$ Haplotypes with frequency $<0.05$ were excluded.

Table 5. Fugl-Meyer and Barthel index scores after treatment with VEGF genes 3025020 and rs3025039.

\begin{tabular}{lccc}
\hline Variable & Genotype & Fugl-Meyer score & Barthel score \\
\hline rs3025020 & TT & $34.6 \pm 5.2$ & $34.8 \pm 4.2$ \\
& CT + CC & $33.6 \pm 6.1$ & $35.2 \pm 6.1$ \\
rs3025039 & TT & $58.5 \pm 5.2$ & $62.3 \pm 4.7$ \\
& CT + CC & $64.6 \pm 6.6^{*}$ & $70.5 \pm 5.2^{*}$ \\
\hline
\end{tabular}

*Associated with TT of rs3025020 and rs3025039, $\mathrm{P}<0.01$.

The results revealed a significant association between the rs3025020 genotype distribution and patients with $\mathrm{HCH}(\mathrm{P}=0.001$, after Bonferroni's corrections). The patients with $\mathrm{HCH}$ had a significantly higher frequency of the $\mathrm{T}$ allele $(\mathrm{P}=0.0002, \mathrm{OR}=1.619,95 \% \mathrm{CI}=$ 1.256-2.088). Compared with the healthy controls, the $\mathrm{CC}$ homozygotes of rs3025039 were significantly overrepresented in the patients with $\mathrm{HCH}(\mathrm{P}=0.005)$. The patients with $\mathrm{HCH}$ had a significantly higher frequency of the rs $3025039 \mathrm{~T}$ allele $(\mathrm{P}=0.001, \mathrm{OR}=1.682,95 \% \mathrm{CI}$ $=1.246-2.270$ ).

In block 3 , significantly more C-G-C (rs3025020, rs3025030, and rs3025039) haplotypes $(\mathrm{P}=0.001)$ were found in the controls. Moreover, significantly more T-G-C haplotypes $(\mathrm{P}=0.046)$ were found in the patients with $\mathrm{HCH}$ as compared to the controls. 


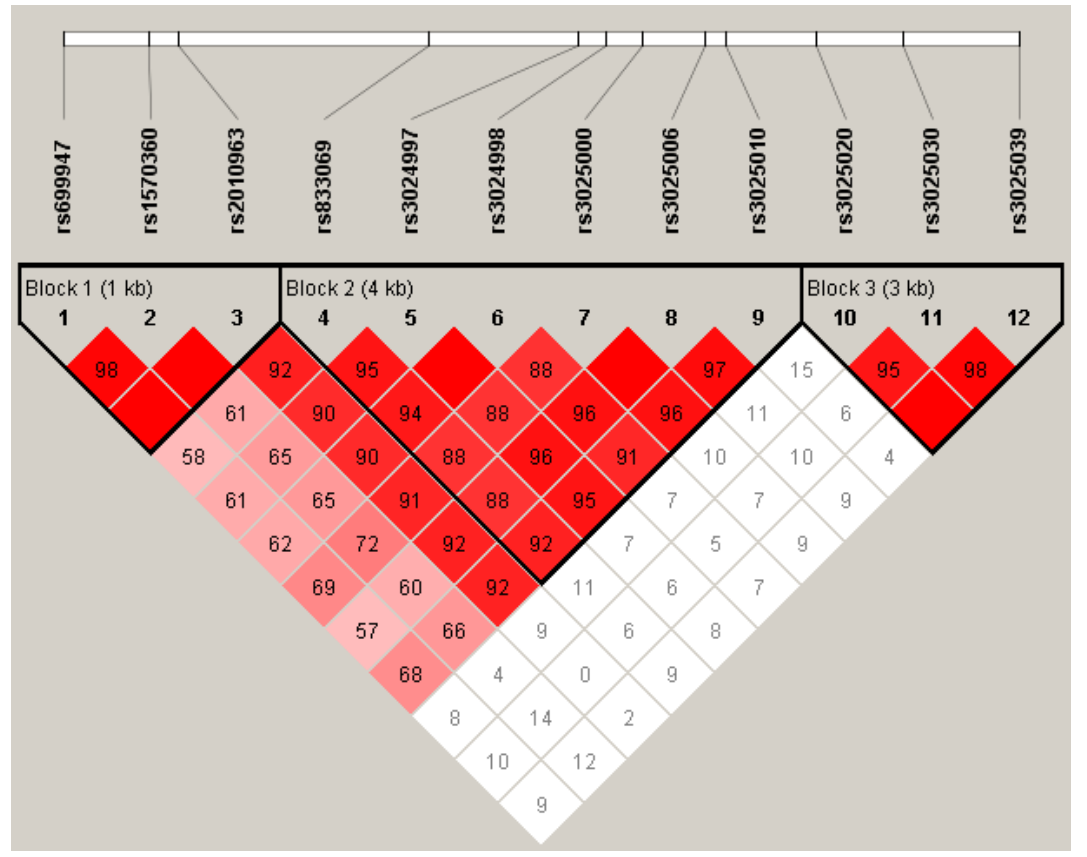

Figure 1. LD plot of the 12 SNPs in the $V E G F$ gene. Values in squares are the pairwise calculation of $\mathrm{r}^{2}$. Black squares indicate $\mathrm{r}^{2}=1$ (i.e., perfect LD between a pair of SNPs). Empty squares indicate $\mathrm{D}^{\prime}=1$ (i.e., complete LD between a pair of SNPs).

Further genotype and clinical phenotype correlations of the rs3025039 carriers showed that Fugl-Meyer and Barthel index scores were lower in the patients with the TT genotype relative to $\mathrm{CT}+\mathrm{CC}$ genotypes $(\mathrm{P}<0.01)$.

\section{DISCUSSION}

$\mathrm{HCH}$ is a very complex disease. Its progression is multifactorial and thus influenced by genetic and environmental factors. In this study, we examined VEGF as a potential disease susceptibility marker for $\mathrm{HCH}$, as well as a rehabilitative treatment in a Chinese Han population. Our results provide direct evidence that $V E G F$ polymorphisms are linked to $\mathrm{HCH}$ and rehabilitative treatment, and extend the list of variants that may affect the development of HCH (Schneider et al., 2008; Hamedian et al., 2012).

We showed here in a cohort of the Chinese Han population that VEGF polymorphisms appear to be relevant to $\mathrm{HCH}$. In particular, the frequency of the T allele (rs3025020) is significantly higher in the $\mathrm{HCH}$ subjects relative to controls. Interestingly, Fugl-Meyer and Barthel index scores were lower in the patients with the TT genotype relative to $\mathrm{CT}+\mathrm{CC}$ genotypes. Consistently, previous studies have shown that the VEGF 3'-UTR polymorphism (rs3025039) is associated with other polymorphisms (Ungerback et al., 2009; Etienne-Grimaldi et al., 2011). For $V E G F$ rs3025039, an association between the $C$ allele and higher VEGF plasma levels has been found in healthy subjects (Renner et al., 2000). The association of the VEGF rs3025039 TT genotype with higher hypertension is consistent with the notion that SNPs as- 
sociated with lower VEGF levels are related to a higher hypertension (Morita et al., 2013). The patients with less plasma VEGF levels may be at higher risk for bevacizumab-related hypertension. This notion is consistent with the view that the binding of VEGF to VEGFR leads to vasodilatation and reduced blood pressure (Facemire et al., 2009; Jubb and Harris, 2010).

The most significant issue with $\mathrm{HCH}$ lies in its high disability rate, which is an important factor that affects the patients' quality of life. With motor and linguistic disorders, the patients will experience significant deterioration in quality of life, which burdens the patients, their family, and society. Our experience has demonstrated that early-implemented rehabilitative training produces significant efficacy in improving motor functions of affected limbs and promoting restoration of linguistic functions. Our results indicated that Fugl-Meyer and Barthel index scores were lower in the patients with the rs3025039 TT genotype relative to CT + $\mathrm{CC}$ genotypes. The patients with $\mathrm{HCH}$ will be benefited from rehabilitative therapies (including acupuncture, physical therapy, hyperbaric oxygen therapy, and linguistic communication).

There was a significant between-group difference in the genotype distribution of rs3025020. Specifically, HCH subjects had a significantly higher frequency of the T allele of rs3025020. To our knowledge, our study is the first study to identify a significant association between rs3025020 in the intron 6 region of the $V E G F$ gene and $\mathrm{HCH}$.

We further investigated the interaction among polymorphisms and observed strong LD. The haplotype analysis revealed that significantly more T-G-C haplotypes were found in the patients with $\mathrm{HCH}$. These results indicated that the patients with T-G-C (block 3) haplotypes of the $V E G F$ gene were more prone to $\mathrm{HCH}$. Moreover, significantly more C-G-C haplotypes were detected in the healthy controls than in the patients with $\mathrm{HCH}$, suggesting that they may show protective effects against $\mathrm{HCH}$. To some extent, this finding further supports a role of $V E G F$ polymorphisms in $\mathrm{HCH}$, while an ethnic group difference may exist.

In conclusion, our study suggests a potential role of the VEGF SNPs and their related haplotypes in the genetic susceptibility to $\mathrm{HCH}$ and rehabilitative treatment. Further studies are needed to investigate how these SNPs affect the function of VEGF. A broader examination of the genetic variation in VEGF in the Chinese Han population may reveal other variants associated with disease risk.

\section{Conflicts of interest}

The authors declare no conflict of interest.

\section{ACKNOWLEDGMENTS}

Research partially supported by the Genetic Resource of Chinese population (\#57004).

\section{REFERENCES}

Advani A, Kelly DJ, Advani SL, Cox AJ, et al. (2007). Role of VEGF in maintaining renal structure and function under normotensive and hypertensive conditions. Proc. Natl. Acad. Sci. U. S. A. 104: 14448-14453.

Belgore FM, Blann AD, Li-Saw-Hee FL, Beevers DG, et al. (2001). Plasma levels of vascular endothelial growth factor and its soluble receptor (SFlt-1) in essential hypertension. Am. J. Cardiol. 87: 805-807.

Blumberg HP, Wang F, Chepenik LG, Kalmar JH, et al. (2008). Influence of vascular endothelial growth factor variation on human hippocampus morphology. Biol. Psychiatr. 64: 901-903. 
Brogan IJ, Khan N, Isaac K, Hutchinson JA, et al. (1999). Novel polymorphisms in the promoter and 5'-UTR regions of the human vascular endothelial growth factor gene. Hum. Immunol. 60: 1245-1249.

Celletti FL, Waugh JM, Amabile PG, Brendolan A, et al. (2001). Vascular endothelial growth factor enhances atherosclerotic plaque progression. Nat. Med. 7: 425-429.

Dahlberg SE, Sandler AB, Brahmer JR, Schiller JH, et al. (2010). Clinical course of advanced non-small-cell lung cancer patients experiencing hypertension during treatment with bevacizumab in combination with carboplatin and paclitaxel on ECOG 4599. J. Clin. Oncol. 28: 949-954.

De Stefano A, Carlomagno C, Pepe S, Bianco R, et al. (2011). Bevacizumab-related arterial hypertension as a predictive marker in metastatic colorectal cancer patients. Cancer Chemother. Pharmacol. 68: 1207-1213.

Etienne-Grimaldi MC, Formento P, Degeorges A, Pierga JY, et al. (2011). Prospective analysis of the impact of VEGF-A gene polymorphisms on the pharmacodynamics of bevacizumab-based therapy in metastatic breast cancer patients. Br. J. Clin. Pharmacol. 71: 921-928.

Facemire CS, Nixon AB, Griffiths R, Hurwitz H, et al. (2009). Vascular endothelial growth factor receptor 2 controls blood pressure by regulating nitric oxide synthase expression. Hypertension 54: 652-658.

Ferrara N and Davis-Smyth T (1997). The biology of vascular endothelial growth factor. Endocr. Rev. 18: 4-25.

Hamedian AA, Esteghamati A, Noshad S, Mozafari M, et al. (2012). Vascular endothelial growth factor (VEGF) +405 $\mathrm{C} / \mathrm{G}$ polymorphism is associated with essential hypertension in a population from Tehran of Iran. Mol. Biol. Rep. 39: 6213-6218.

Jain L, Sissung TM, Danesi R, Kohn EC, et al. (2010). Hypertension and hand-foot skin reactions related to VEGFR2 genotype and improved clinical outcome following bevacizumab and sorafenib. J. Exp. Clin. Cancer Res. 29: 95.

Jubb AM and Harris AL (2010). Biomarkers to predict the clinical efficacy of bevacizumab in cancer. Lancet Oncol. 11: $1172-1183$.

Lee HH, Hong SH, Shin SJ, Ko JJ, et al. (2010). Association study of vascular endothelial growth factor polymorphisms with the risk of recurrent spontaneous abortion. Fertil. Steril. 93: 1244-1247.

Loomans CJ, Dao HH, van Zonneveld AJ and Rabelink TJ (2004). Is endothelial progenitor cell dysfunction involved in altered angiogenic processes in patients with hypertension? Curr. Hypertens. Rep. 6: 51-54.

Morita S, Uehara K, Nakayama G, Shibata T, et al. (2013). Association between bevacizumab-related hypertension and vascular endothelial growth factor (VEGF) gene polymorphisms in Japanese patients with metastatic colorectal cancer. Cancer Chemother. Pharmacol. 71: 405-411.

Renner W, Kotschan S, Hoffmann C, Obermayer-Pietsch B, et al. (2000). A common 936 C/T mutation in the gene for vascular endothelial growth factor is associated with vascular endothelial growth factor plasma levels. J. Vasc. Res. 37: 443-448.

Rini BI, Cohen DP, Lu DR, Chen I, et al. (2011). Hypertension as a biomarker of efficacy in patients with metastatic renal cell carcinoma treated with sunitinib. J. Natl. Cancer Inst. 103: 763-773.

Schneider BP, Wang M, Radovich M, Sledge GW, et al. (2008). Association of vascular endothelial growth factor and vascular endothelial growth factor receptor-2 genetic polymorphisms with outcome in a trial of paclitaxel compared with paclitaxel plus bevacizumab in advanced breast cancer: ECOG 2100. J. Clin. Oncol. 26: 4672-4678.

Schrijvers BF, Flyvbjerg A and De Vriese AS (2004). The role of vascular endothelial growth factor (VEGF) in renal pathophysiology. Kidney Int. 65: 2003-2017.

Ungerback J, Elander N, Dimberg J and Soderkvist P (2009). Analysis of VEGF polymorphisms, tumor expression of VEGF mRNA and colorectal cancer susceptibility in a Swedish population. Mol. Med. Rep. 2: 435-439. 